\title{
Au pays des supplices : caricature et paroxysme dans la Chine fantasmée d'Octave Mirbeau
}

La postérité a surtout retenu d'Octave Mirbeau deux romans, publiés à un an de distance : Le Jardin des supplices ${ }^{1}$ et Le Journal d'une femme de chambre ${ }^{2}$. Si le second a connu une fortune largement publique, le premier conserve une notoriété marquée par le soufre. Cependant, l'un et l'autre visent à bouleverser l'ordre social établi et la morale bourgeoise, dont l'hypocrisie consubstantielle est aux yeux d'Octave Mirbeau le masque de toutes les infamies. Les deux romans, dont le dispositif d'écriture diverge à peine, le premier affectant la forme du journal intime, l'autre de la confession, s'opposent à première vue par leur cadre : le Journal brocarde la bourgeoisie provinciale, tandis que le Jardin met en scène la vie oisive et sulfureuse d'une riche expatriée anglaise et de son amant français à Canton. Le Jardin des supplices est à la fois le titre du roman et de sa deuxième partie, qui suit un premier récit, «En Mission ", lui-même précédé d'un frontispice, les deux premiers segments préparant le paroxysme narratif du dernier. L'ensemble, discontinu plus que disparate, emprunte son titre au bagne supposé de cette ville, épicentre du roman, lieu où s'incarnent les fantasmes érotiques, sadiques et macabres de l'héroïne Clara, lesquels s'illustrent dans une Chine dont la représentation est non moins hallucinée que ne l'est la conscience de la protagoniste, prisonnière d'une frénésie sexuelle où « petite mort » et trépas viennent à se confondre. Cet article se propose d'étudier les moyens et stratégies de la caricature dans l'une des œuvres emblématiques du discours européen sur la civilisation chinoise dans la première moitié

Gaultier Roux - maître de conférences, Université Fudan, Faculté des Langues et Littératures Étrangères, Département de Français. Adresse de correspondance : Humanities Building 311, Guonian Road 299, Yangpu District, Shanghai 200433, China ; e-mail : gaultierroux@fudan.edu.cn

ORCID iD : https://orcid.org/0000-0003-4725-756X

1. Mirbeau, O. (1899). Le Jardin des supplices. Paris : Charpentier-Fasquelle.

2. Mirbeau, O. (1900). Le Journal d'une femme de chambre. Paris: Charpentier-Fasquelle. 
du vingtième siècle. Son objectif sera d'examiner comment cette représentation, loin de jeter un regard réaliste sur la Chine, vise au contraire, une fois libérée de la nécessité de la mimésis et usant à loisir de l'exacerbation verbale et figurative, à y projeter une caricature en creux de son pendant, l'Europe.

\section{Une Chine stylisée à l'extrême}

La critique a peu relevé, jusqu'à ce jour, le contexte dans lequel la représentation de la Chine s'inscrit lorsque Mirbeau, à compter de mai 1896, publie dans la presse les textes qui seront les éléments constitutifs du roman, selon un procédé de réécriture et d'amplification qui caractérise son œuvre ${ }^{3}$. Certes, le roman s'inscrit dans les polémiques de l'Affaire Dreyfus (Glaudes, 2018), et de ce fait on doit y lire une critique implicite de l'autorité politique, militaire, religieuse et de l'institution judiciaire. Mais ce roman s'inscrit aussi dans le prolongement de l'apogée et de la remise en question des Empires coloniaux, empires qui ont donné naissance à deux traditions littéraires divergentes, celle de l'exotisme nostalgique d'une part et celle des récits d'aventure exaltant l'épopée coloniale d'autre part, que Mirbeau met ici toutes deux à distance (v. Ducrey et Moura, 2002, p. 9-19). Il faudrait ajouter, sans minimiser l'influence du climat national sur l'écriture du roman, que l'œuvre s'inscrit à part égale au moins dans un nouveau contexte géopolitique qui déplace et renouvelle la question coloniale. En effet, le Japon émerge alors sur la scène internationale et traite désormais à parité avec les puissances occidentales traditionnelles, alors que l'agonie de l'Empire chinois excite les luttes d'influence. L'apparition du péril jaune est concomitante de la rédaction du roman : l'expression s'impose dès 1895 après la publication d'un article relatif à la reproduction d'une allégorie caricaturale d'Hermann Knackfuss, d'après une esquisse de l'empereur Guillaume II (Poulet, 2002, p. 53) : Die Gelbe Gefahr. Cette allégorie d'un académisme on ne peut plus kitsch illustre l'essentialisme triomphant et la conscience institutionnalisée de la supériorité des races, à une époque où le darwinisme extrapolé à la politique autorise tous les raisonnements et s'illustre à plein dans l'anticipation d'un choc des civilisations. Dès 1897, le sociologue Jacques Novicow publie un opuscule par lequel il démontre le caractère infondé de cette psychose, non sans ironiser sur la puissance supposée d'un Empire chinois à son déclin. Ce n'est toutefois qu'après la révolte des Boxers (1899-1901), et plus encore après la guerre russo-japonaise (1904-1905) que l'expression sera pleinement consacrée. En France, à la même époque, Edmond Théry fait de l'antagonisme racial la cause inéluctable d'un combat pour la survie de la race blanche. Le fantasme culmine assurément en France en 1908 avec la trilogie d'Émile Driant, L'Invasion jaune.

3. Les premières pages du Frontispice paraissent dans Le Journal le 31 mai 1896, sous le titre «Divagations sur le meurtre». 
On voit comment donc le roman de Mirbeau prend pied parmi les illustrations littéraires du péril jaune, et cependant il en est assurément la contre-illustration, non qu'Octave Mirbeau prenne la défense des Chinois, mais qu'il dénigre systématiquement les prétentions au caractère moral des civilisations : dans un article publié en 1901, "Sur un vase de Chine ${ }^{4}$, l'auteur utilise le terme «barbare » dans un sens sarcastique qui dénonce la posture occidentale. Et de la même manière dans Le Jardin des supplices, Mirbeau oppose, pour mieux les confondre, l'une à l'autre les civilisations occidentale et orientale, identiquement violentes, corrompues et corruptrices. Participant d'une rhétorique de l'exubérance qui est d'abord celle d'une pleine jouissance dans l'acte d'écriture, ce récit bannissant les censures se complaît dans l'abondance des descriptions suffocantes et malignes d'une nature profuse jusquà l'irréalisme et d'un goût du sang et de la souffrance qui caractérise l'humanité plus que les Chinois. Influencé notablement par Huysmans, dont $\grave{A}$ rebours a fait date en 1884, ce récit transpose les pulsions paroxystiques de la société de la Belle-Époque et, en particulier, de l'esthétique décadente. La Chine que dépeint Mirbeau est un espace à la fois minimal et séminal. Minimal en premier lieu, parce qu'il se singularise par la restriction extrême du décor. Les éléments d'ensemble demeurent conventionnels et leur caractérisation faible : ce paradigme structurel indique assurément une parenté avec le roman libertin dont le dispositif romanesque élude le réalisme. Chez Mirbeau, de fait, la rareté des toponymes (Canton), des anthroponymes (la batelière Ki-Paï), l'extrême restriction de champ des paysages (le bagne et la rue aux bouchers, le palais des plaisirs de l'autre côté de la rivière et le sampang qui y transporte) déjouent la reconstitution mimétique de la Chine. Toutefois, on distingue une double opération dans ce dispositif scénographique : d'une part l'auteur déjoue l'esthétique de la "Chine de paravent (Loti, 1906, p. 268) au pittoresque artificiel et mièvre, d'autre part il abstrait le texte d'une contextualisation restrictive, dégageant la lecture d'un quelconque pacte de sincérité. La Chine est ainsi réduite à un certain nombre de marqueurs suggestifs (la référence littéraire à Yu Sin ${ }^{5}$, à la monnaie - taëls -, à la faune - grues, hirondelles, gecko) ou plus largement exotiques (le climat subtropical, la consommation de bétel, et dans une proportion inégalée, la flore). Dans la prolongation du japonisme de l'époque, on trouve parmi les marqueurs des objets rares, délicats ou insolites, comme le coffret destiné à recueillir les instruments du bourreau : « La boîte en était charmante et d'un laque admirable : un vol d'oies sauvages, au-dessus d'un étang nocturne où la lune argentait les lotus et les iris ». (Mirbeau, 1991, p. 204).

4. Le Journal, 4 mars $1901: 1^{\mathrm{e}}$ page.

5. Poète, homme politique des dynasties Liang et Zhou du Nord (513-581), co-fondateur d'un style particulier et maître du fù, poème rhapsodique en prose. Cette référence (p. 182) est associée à celle de Robert de Montesquiou qu'elle côtoie immédiatement : ce dispositif souligne le double référent du roman : à savoir son référent littéral, la Chine intemporelle, et son référent figuratif, l'Occident contemporain. 
Le lieu dépeint est également symbolique, en ce que, réduit à l'extrême, il devient suggestif d'une extrapolation, voire d'une universalisation dans une rupture déchelle qui est assez caractéristique de l'esprit scientifique du temps : au microcosme de l'espace du bagne correspond le macrocosme de l'humanité. L'appellation " jardin des supplices " renvoie par antithèse au jardin édénique, dont il subvertit les codes mythologiques. Plus qu'une réécriture de la Genèse, Mirbeau se déplace vers une opération de diffraction de références à la manière du triptyque de Jérôme Bosch ${ }^{6}$ lequel, une fois refermé, représente un monde initial plat et circulaire dont la forme correspond à celle du jardin de Mirbeau. Il faut relever l'analogie dantesque comme jeu intertextuel : non seulement la géographie par imbrication reproduit dans une moindre mesure les bolges infernales, mais la segmentation de l'espace en deux opposés de structure analogue mais renversée (le bagne et, de l'autre côté de la rivière, le palais des plaisirs) rappelle Enfer et Paradis dans une double perversion des modèles : l'Enfer est désormais le lieu de la torture arbitraire où le supplicié n'est pas nécessairement coupable, le Paradis est le lieu d'une félicité orgasmique et frénétique. Entre imitation et déformation, Mirbeau procède ainsi en filigrane à une réécriture de modèles occidentaux dont il détourne les caractéristiques fondamentales pour signaler la charge qu'il opère. De la même manière, Mirbeau subvertit les codes de la vraisemblance romanesque qui ont été érigés en système de représentation depuis le début du siècle : le roman, délibérément délirant, crée une utopie paradoxale. On assiste alors à un déplacement de la quête de vérité, d'une reconstitution du monde vers un discours sur celui-ci. Alors qu'Auerbach (1969) avait relativisé, dans le processus de mimésis, l'importance du détail pour préciser le critère de la foi donnée au texte, on observe à quel point chez Mirbeau l'Ansatzpunkt minimal est opérant, déplaçant l'objet du roman d'une vérité ou d'une vraisemblance des faits vers la vérité du langage.

\section{La Chine, lieu du paroxysme}

La Chine devient ainsil l'espace mental d'une expérience de pensée dans lequel Mirbeau peut donner cours à une critique acerbe du champ politique et du champ artistique. En ce sens, on pourrait opter pour une lecture parodique du Jardin des supplices, qui pourrait être considéré comme un pastiche, voire en partie un autopastiche, du goût fin-de-siècle et du décadentisme littéraire. Non seulement le traitement du végétal reproduit celui d'un Huysmans dans À rebours ou de Zola (v. Roy-Reverzy, 1997), mais d'évidence le narrateur, efféminé par sa maitresse au contraire virilisée, reproduit l'archétype du mâle fin-de-siècle qu'on retrouve dans La Femme et le Pantin de Pierre

6. Bosch J. (ca. 1494 - 1506), Le Jardin des délices, Madrid, Musée du Prado ( ${ }^{\circ}$ d'inventaire P002823) : notons que le titre, apocryphe, est fixé à cette même époque par les historiens de l'art. Voir Przybos (1992). 
Louÿs (1898). À la différence de ces deux références, on note chez Mirbeau un goût du grotesque qui accentue la distance ironique de l'auteur à son œuvre, et rappelle l'exagération parodique d'un Adoré Floupette putatif dans Les Déliquescences de Gabriel Vicaire et Henri Beauclair (1885). En ce sens le récit romanesque déplace une partie de son enjeu vers une lecture distanciée du Zeitgeist dont Mirbeau reproduit les codes pour mieux en déjouer la facticité, non sans, et c'est la complexité de ce récit, en jouir par l'exercice de style qu'il propose et la fantaisie absolue que permet la référence à la Chine.

Mirbeau redouble ainsi le topos contemporain de la suffocation par le végétal par une suffocation d'ordre psychologique, qui est celle de l'insoutenable spectacle, relevée par Georges Bataille (1987, p. 42). Les deux sont de l'ordre de l'expérience sensorielle, mais la torture, à la différence de la végétation, est spécifiquement scopique et s'inscrit dans un schème voyeuriste qui pose la question de la posture éthique du touriste dans les pays étrangers. On note ainsi la double analogie avec le jardin zoologique et la visite touristique dans laquelle le voyageur est un spectateur détaché des enjeux de ce qu'il observe (Decome, 2014, p. 218-219). Le but poursuivi par Clara dans son expatriation, c'est une liberté individuelle absolue, et donc littéralement anarchique :

En Chine, la vie est libre, heureuse, totale, sans conventions, sans préjugés, sans lois... pour nous, du moins... Pas d'autres limites à la liberté que soi-même... à l'amour que la variété triomphante de son désir... L'Europe et sa civilisation hypocrite, barbare, c'est le mensonge. (Mirbeau, 1991, p. 133)

On voit ici les niveaux critiques se superposer et rendre la lecture du Jardin des supplices plus complexe que la lecture subversive et amoraliste ne le suggère. Célébration paroxystique de la croissance, de l'excroissance du végétal ou des floraisons humaines de la torture que permet l'exotisme effréné du cadre chinois, le récit laisse libre cours aux fantasmes, sexuels ou macabres, de l'Européen : le fantasme totalisateur de l'exotisme trouve ici sa plus haute parodie.

La Chine est ainsi, pour risquer la métaphore, un laboratoire mental dans lequel le botaniste Mirbeau se double d'un anatomiste pour lequel le corps devient prépotent. Mirbeau expose les corps et les dénude au-delà du possible : ôter la chair du corps, dépecer, c'est encore déshabiller l'humain pour en révéler la macabre vérité (Margat, 2005). Le corps, principal objet de la caricature, est ici travaillé par le bourreau comme par l'auteur : omniprésent, il subit toutes les transformations possibles, dans une jubilation de l'excès qui suscite tant l'horreur que le sentiment d'un burlesque macabre qui se distingue de la posture sadienne proprement dite ${ }^{7}$ et tend vers un registre grand-guignolesque assumé mais dont l'adaptation théâtrale par Pierre Chaine et André de Lorde en 1922 dénature le propos burlesque. L'adapta-

7. Rappelons que l'auteur ne peut avoir accès au répertoire des tortures des Cent vingt jours de Sodome : le roman de Sade demeure inédit jusqu'en 1904. 
tion théâtrale signale cependant la dimension éminemment visuelle du roman et des déformations du corps humain qu'il propose ${ }^{8}$.

La corporéité s'inscrit à plein dans le projet mirbellien : roman du corps sentant, il est aussi roman du corps ressenti. On peut prendre pour exemple le corps dédoublé du Chinois, à la fois bourreau et victime : l'anonymat des personnages exhibe l'indifférenciation des identités. Victime et bourreau sont assimilés par leur laideur commune ; ce qui les distingue, et les oppose par pur artifice, c'est la nature même du démembrement qu'on opère : pour le bourreau obèse, le «patapouf » de Clara, la dilacération est visuelle et s'effectue à travers le regard sardonique de l'Occidentale perverse ; tandis que pour la victime, la dilacération, l'atomisation du corps s'effectue par l'opération réputée savante du bourreau farcesque. Cil et couteau font le même ouvrage. Or dans cette double caricature du type chinois, nulle physiognomonie : les corps ne révèlent rien du caractère, des inclinations, en somme, de la nature humaine du sujet. Au contraire, ils sont indifférents, ne recèlent aucune signification propre, ne sont que de purs phénomènes. Sur ces corps, l'auteur procède par deux régimes d'amplification, le premier étant celui du portrait-charge, traversé par des processus d'amplification qui déforment les corps jusqu'au ridicule et au déplaisant ; le second étant offert par la torture restituée par une scrupuleuse minutie stylistique, hyperbolique et hyperréaliste, poussée jusqu'à l'horreur grotesque de visions cauchemardesques que rendent les eaux fortes de Raphaël Freida (fig. 1). Le bourreau devient ainsi l'anti-Pygmalion ${ }^{9}$ qui suscite, par son art, non la conjonction de la beauté et de la vie, mais de la laideur et de la mort, considérées comme une forme antithétique de beauté. Loin d'appeler la vie, l'acte créateur appelle au contraire l'anéantissement.

Fig. 1. Eaux-fortes de Raphaël Freida. Planche hors-texte (cliché coll. part). Dans O. Mirbeau (1927). Le Jardin des supplices. Paris : Javal et Bourdeaux ${ }^{10}$.

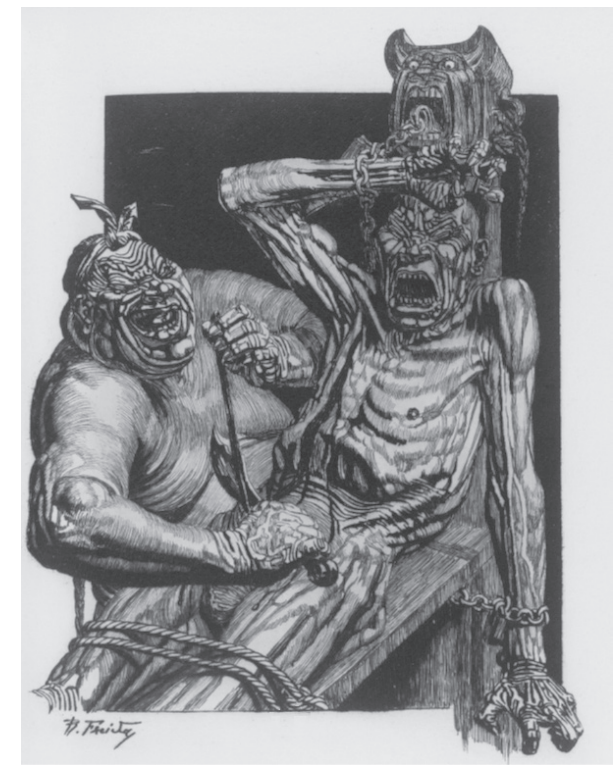

8. L'iconicité et la plasticité du récit sont encore soulignées par la commande d'A. Vollard à Rodin, lequel lui livre vingt compositions originales pour un volume illustré (1902) et l'édition Javal et Bourdeaux accompagnée de douze eaux-fortes de Raphaël Freida (1927).

9. L'allusion est sensible : «C'est-à-dire travailler la chair humaine, comme un sculpteur sa glaise ou son morceau d'ivoire [...]». p. 206.

10. "J'ai retaillé un homme, des pieds à la tête, après lui avoir enlevé toute la peau » (Mirbeau, 1991, p. 202). La référence au supplice du lingchi est évidente. Voir Decome (2014) pour une analyse de la réception de cette torture en France). 


\section{Une esthétique du déplacement}

Le roman mirbellien s'inscrit dans une triple transgression : de la fiction, de la morale, et du bon goût. Le déplacement spatial et culturel permet de dénoncer la tartuferie occidentale. Le discours offre un clair retournement par la bouche du bourreau : " Le snobisme occidental nous envahit, les cuirassés, les fusils à longue portée, l'électricité, les explosifs... que sais-je ? tout ce qui rend la mort collective, administrative et bureaucratique... » (Mirbeau, 1991, p. 207). Par ce contre-discours, Mirbeau illustre les conséquences du triomphe de l'esprit bourgeois : industrialisation de la guerre, massification de leur effet, et au-delà, mondialisation rampante qui homogénéise le monde. Mais loin de proposer un contre-modèle, car en bon sceptique il ne saurait y adhérer, l'imprécateur accomplit son rôle qui est de tendre à son interlocuteur le miroir dans lequel ce dernier prendra la mesure du réalisme symbolique de la caricature. L'universelle caricature est soulignée par Clara : «Évocation grotesque et sinistre du progrès occidental, un pasteur - seul être humain - y rôdait [...: : il avait conservé, comme dans les brumes métropolitaines, son caricatural uniforme de clergyman $[\ldots] »($ p. 192). La satire diffractée de l'Europe fait donc œuvre dans ce viol de la conscience, cette pénétration par effraction que le récit opère dans le jugement du lecteur ; le caractère formellement scandaleux du texte est ainsi le moyen résolument sadien de provoquer un dessillement du lecteur. Ce faisant, Mirbeau assume une charge satirique qui se libère dans son paroxysme, la nécessité comique : au-delà de l'outrance ne demeure que le rire sardonique. En cela, il emprunte à Barbey d'Aurevilly son sens radical du geste caricatural : « La caricature, c'est l'outrance d'une vérité, d’une vérité déformée et outragée, mais cependant visible encore. La caricature, c'est le crapaud recherché et retrouvé, par un art retors et humiliant, dans le pur profil d'Apollon.» (1883, p. 287).

Indéniablement, Le Jardin des supplices pose un problème de lecture, et représente même un cas exemplaire de réception biaisée, comme en témoignent les œuvres qui en sont directement inspirées (Thévenin, 1906) ou les adaptations (Chaine et Lorde en 1922 pour le théâtre, Christian Gion pour le cinéma en 1976, ou encore de M. Brice dans La Dame de Shanghaï, dans la série «Brigade mondaine » en 1993). Une lecture par trop orientale, ou orientaliste, tend à voir dans la Chine mirbellienne une représentation d'une Chine supposée réelle, comme si la projection du sujet occidental devenait une vérité substituable au fait. La portée ironique fondamentale du propos, qui repose sur un jeu de déplacement familier aux lecteurs de contes philosophiques, est parfois occultée, notamment par la puissance érotique et sadique que l'auteur a su développer dans l'ouvrage. Malgré la vigueur du trait et l'acidité des propos, malgré la violence des images, le jeu d'écriture repose fondamentalement sur une distance ironique qui déjoue la valeur accusatrice de la caricature du type Chinois ; ici, la mise en scène, l'exhibition qui ressortissent de la vis comica traditionnelle sont détournées par la souffrance qu'elles sous-tendent, et l'horreur fascinée qu'elles suscitent, horreur 
dont Clara et le narrateur représentent les deux pôles : la fascination et la répulsion, qui, ni l'une ni l'autre, n'échappent à leur magnétisme scopique. L'on ne peut se retenir de voir, ni par volupté, ni par répugnance ${ }^{11}$. La visualité tient tant au voyeurisme, dans lequel pulsions érotiques et thanatiques se répondent et correspondent, qu'au principe même du comique, et plus précisément à la gestualité farcesque dont le registre préfigure historiquement la caricature. Or comme la farce, la caricature produit une rétention du sens positif, pour n'exhiber qu'un sens négatif : dans cette perspective, le dispositif convient parfaitement à Octave Mirbeau qui n'entend nullement proposer une morale substitutive à l'absence de morale que recouvre la prétention au contraire des sociétés organisées.

Deux niveaux de lecture se dégagent dans le dispositif ironique mis en place par Mirbeau : le premier est celui du corps mis en scène, dévoilé par sa nudité, rendu abject par sa laideur et sa crasse, exhibé jusqu'au dépècement, dans une forme de jeu ludique d'écriture qui est celui du jeu de la limite du dicible et de l'indicible. Ce niveau est celui du ridicule, du grotesque, celui du premier contact, de la première vue : il y a une agression lors de la lecture, dont on doit saisir en même temps la violence cynique. Mais à ce premier niveau se superpose un second niveau de lecture, plus participatif que le premier, est qui est celui du jeu ironique, lequel crée des effets de disjonction qui désorientent la lecture en ce qu'elles décontextualisent une Chine de convention pour produire une critique distanciée de la représentation stéréotypée de l'altérité dans laquelle se dégage le véritable discours, maintenu dans l'implicite, d'une critique cynique de l'humanité universalisante propagée par l'Europe et de ses prétentions platoniciennes au beau, au bien, au vrai.

L'ironie prend dès lors pour cible le lecteur naif, que la lecture exclusivement exotique du roman dupe. Ainsi l'erreur est-elle manifeste dans la lecture postcoloniale du roman que fait Gianna Quach (1995). Cette lecture critique ne saisit pas la "scène ironique " configurée par l'auteur : dans une configuration actancielle, on voit comment une lecture peut en grande part devenir naïve. On peut extrapoler la scène ironique telle que Philippe Hamon la conçoit à un niveau extradiégétique et structurel (1996, p. 124) : nous obtenons une configuration (fig. 2) qui illustre la visée ironique du roman. Le Chinois n'est pas l'ironisé, mais l'objet du jeu ironique.

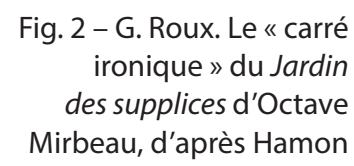

(1996), p. 124.

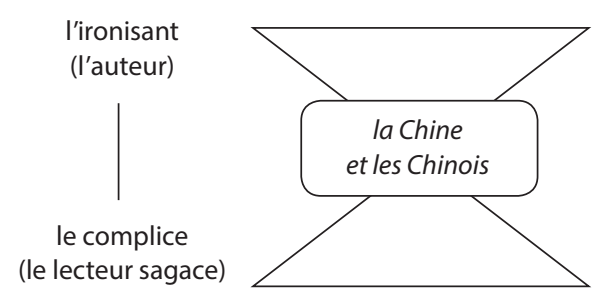

l'ironisé (le « bourgeois ») (le lecteur ingénu)

11. On renverra le lecteur à la lecture de Georges Bataille (1987) et de Julia Kristeva (1980, p. 9) notamment sur le phénomène de l'abjection. 
À travers une fiction de la Chine s'arc-boutant à l'extrême sur les stéréotypes discursifs occidentaux, et les développant dans une caricature outrancière, l'ironisant vise à délégitimer la conscience bourgeoise, à la fois potentiellement scandalisée par le discours du roman, mais pourtant sinon autrice, au moins propagatrice de cette représentation stéréotypée de l'altérité, largement raciale et xénophobe. Le lecteur sagace, complice de l'auteur, comprend le double niveau du texte, à savoir son niveau caricatural et son niveau ironique, dans lequel l'ironisé est composé par la cohorte des dédicataires de l'œuvre : "Aux Prêtres, aux Soldats, aux Juges, aux Hommes qui éduquent, dirigent, gouvernent les hommes [...] " (Mirbeau, 1991, p. 41).

Si Le Jardin des supplices doit être considéré comme un récit de l'altérité, c'est non tant parce qu'il dépeint une Chine qui, on l'a démontré, relève à la fois du conte philosophique et de la caricature, mais parce qu'il dépeint fondamentalement l'altérité en soi, à savoir la pulsion de mort dont le frontispice est le sujet. Récit de jouissance, et en premier lieu du plaisir même d'écrire, l'œuvre déplace son enjeu à un niveau rhétorique dans lequel la relation de la fiction au réel n'est pas une relation de représentation, mais de commentaire. Et de la puissance inhérente au geste caricatural, Mirbeau retient avant tout l'ambition de ridiculiser le sérieux et rendre sérieux le ridicule : l'esthétique de l'inversion, des inversions, joue à plein.

Mirbeau fait donc bien plus que de caricaturer une Chine : il tend au contraire un miroir à l'Europe, et c'est là son tour de force. Sous le Chinois, l'Européen : non seulement il confronte l'Européen à l'hypocrisie de son hédonisme qui avance masqué par la morale, mais il le confronte aussi au paradoxe qui le conduit à désirer ce qu'il réprouve, jusqu'à la limite des sens, révélant la part sadique, sinon masochiste également, de la civilisation européenne, incarnée tour à tour dans le personnage exalté de Clara, riche britannique, et dans la virilité incertaine du narrateur anonyme, petit escroc français. Il y a surtout chez Octave Mirbeau, outre la jouissance de l'écriture, toujours sensible, une contre-jouissance : celle de la dérision, celle de tromper le lecteur naif qui prendra le texte au pied de la lettre, car la caricature mirbellienne est toujours à double fond. La subtilité de Mirbeau trouve une double postérité : celle de la reprise du stéréotype avec Sax Rohmer et son génie du crime, Fu Manchu (treize romans de 1913 à 1959), et celle, ironique et distanciée, de Pierre Boulle (1954). De fait, puisant aux sources des racontars, Mirbeau était devenu l'auteur d'un mythe contemporain dont Le Supplice du santal (2001), par Mo Yan, montre que la littérature chinoise se l'est réapproprié afin de subvertir à son tour le fantasme européen. 


\section{RÉFÉRENCES}

Auerbach, E. (1969) [1946]. Mimésis : la représentation de la réalité dans la littérature occidentale. Paris : Gallimard.

Barbey d'Aurevilly, J. (1883). Les Ridicules du temps : " Les Blagueurs en littérature ». Paris : É. Rouveyre et G. Blond.

Boulle, P. (1954). Le Bourreau. Paris : Julliard.

Bataille, G. (1987). Les Larmes d'Éros. Euvres complètes, X. Paris : Gallimard.

Bernier, L. (1995). L'Imaginaire chinois chez Octave Mirbeau. Dans The Force of Vision. Tokyo : International Comparative Literature Association, II, 448-455.

Chabrier, C. (2006). Aesthetic Perversion: Octave Mirbeau's Le Jardin des supplices. NineteenthCentury French Studies, 4 (3/4), 355-370.

Decome, M. (2014). La Formation du discours conventionnel français sur les Chinois : une approche littéraire, 1840-1945. [Thèse de doctorat. Université Montpellier-III].

Driant, É. (C ${ }^{\text {ne }}$ Danrit). (1908). L'Invasion jaune. Paris : Flammarion.

Ducrey, G. et Moura, J.-M. (éd.). (2002). Introduction. Dans Crise-fin-de-siècle et tentation de l'exotisme (p. 9-19). Lille : Presses de l’Université Lille-3.

Glaudes, P. (2018). Entre diatribe et allégorisme satirique : l'affaire Dreyfus dans Le Jardin des supplices et Le Journal d'une femme de chambre. Studi Francesi, 185, 206-215. https:// journals.openedition.org/studifrancesi/12553

Hamon, Ph. (1996). L'Ironie littéraire. Essai sur les formes de l'écriture oblique. Paris : Hachette.

Kristeva, J. (1980). Pouvoirs de l'horreur. Paris : Seuil.

Loti, P. (1906) [1902]. Les Derniers jours de Pékin. Euvres Complètes, VI. Paris : CalmannLévy.

Margat, C. (2005). Supplice chinois in French Literature: From Octave Mirbeau's Le Jardin des supplices to George Bataille's Les Larmes d'Éros. http://turandot.chineselegalculture. org/Essay.php?ID=38

Mirbeau, O. (1991). Le Jardin des supplices. Paris : Gallimard.

Mo, Y. (2006) [2001]. Le Supplice du santal (Ch. Chen-Andro trad.). Paris : Seuil.

Novicow, J. (1897). Le Péril jaune. Paris : V. Giard et E. Brière.

Poulet, R. (2002). L'Orient : généalogie d'une illusion. Lille : Presses Universitaires du Septentrion.

Przybos, J. (1992). Délices et supplices : Octave Mirbeau et Jérôme Bosch. Dans Octave Mirbeau. Actes du colloque international d'Angers, 19-22 septembre 1991 (p. 207-216). Angers : Presses de l'Université d'Angers.

Quach, G. (1995). Mirbeau et la Chine. Cahiers Octave Mirbeau, 2, 87-100.

Roy-Reverzy, É. (1996). D’une poétique mirbellienne : Le Jardin des supplices. Cahiers Octave Mirbeau, 3, 30-45.

Roy-Reverzy, É. (1997). Les Perversions de la pastorale : La Faute de l'abbé Mouret et Le Jardin des supplices. Littératures, 36, 81-95.

Théry, E. (1901). Le Péril jaune. Paris : F. Juven.

Thévenin, R. (1906). La Cité des tortures. Journal des voyages, $\mathrm{n}^{\text {os }} 518$ à 521.

Zhang, Y. (2002). Octave Mirbeau et la Chine : les paradoxes du jardin exotique. Dans G. Ducrey et J.-M. Moura (éd.), Crise-fin-de-siècle et tentation de l'exotisme (p. 85-100). Lille : Presses de l'Université Lille-3. 


\section{FIGURES}

Freida R. (1927). [Sans titre, planche hors-texte]. Dans O. Mirbeau, Le Jardin des Supplices. Paris : Javal et Bourdeaux.

Roux, G. (2020). Le " carré ironique » du Jardin des supplices d'Octave Mirbeau, d'après Hamon, Ph. (1996). L'Ironie littéraire. Essai sur les formes de l'écriture oblique (p. 124). Paris : Hachette.

RÉSUMÉ : Cet article étudie les stratégies de la caricature dans Le Jardin des supplices, œuvre emblématique du discours exotique et jalon dans la constitution des stéréotypes sur la Chine. Cependant, le récit, loin de chercher à représenter une Chine réaliste, ni même à en indiquer la barbarie supposée, vise au contraire à produire en creux une caricature de son pendant européen. Le Jardin des supplices a ainsi une vocation spéculaire, par laquelle l'Occident se reconnaît dans l'utopie fantasmagorique proposée; on identifiera alors à la fois, dans cette farce sadique, une parodie des structures institutionnelles européennes et un pastiche des traits caractéristiques de la décadence littéraire. Nonobstant, Le Jardin des supplices informe bien durablement, et au second degré, la représentation de la Chine et des Chinois dans l'inconscient collectif.

Mots-clés : caricature, ironie, érotisme, sadisme, exotisme, O. Mirbeau, Chine

\section{In the land of torture: caricature and paroxysm in Octave Mirbeau's fantasized China}

ABSTRACT: This article studies the tools and strategies employed in the creation of caricature in one of the prototypical works of the European exotic discourse. Le Jardin des Supplices is indeed an important milestone in the formation of stereotypes about China; however, this representation, far from seeking to develop a realistic depiction of China, or even to point out its supposed barbarity, aims, on the contrary, at producing a caricature of its counterpart, Europe, while using verbal and figurative exacerbation as main devices. Le Jardin des Supplices acts as a mirror in which the West recognizes itself in the described phantasmagorical utopia; in this sadistic farce, we will therefore identify both a parody of European institutional structures and a pastiche of the characteristic features of literary decadence. Notwithstanding, Le Jardin des Supplices provides a lasting and ironical view of the representation of China and the Chinese in the collective unconscious.

Keywords: caricature, irony, eroticism, sadism, exoticism, O. Mirbeau, China 